\title{
Supervised physical exercise therapy of peripheral artery disease patients: M-health challenges and opportunities
}

\author{
Hugo Paredes, Dennis Paulino, \\ João Barroso \\ Universidade de Trás-os-Montes e \\ Alto Douro (UTAD) and INESC TEC \\ \{hparedes,dpaulino,jbarroso\}@utad.pt
}

\author{
Catarina Abrantes, Isabel Machado \\ CIDESD/Universidade de Trás-os- \\ Montes e Alto Douro (UTAD) \\ \{abrantes,isamachado\}@utad.pt
}

\author{
Ivone Silva \\ Centro Hospitalar e Universitário do \\ Porto and Instituto de Ciências \\ Biomédicas Abel Salazar (ICBAS) \\ heitor.ivone@gmail.com
}

\begin{abstract}
Peripheral artery disease (PAD) main symptom is intermittent claudication, causing pain and limiting the walking abilities of patients, forcing individuals to temporarily stop walking. One treatment advised to counteract the effects of this disease is the practice of physical exercise with monitoring. Currently the monitored exercise programs are applied at the hospital, so some patients have to travel long distances three times a week, with high costs and low adherence of the patients. This paper presents the cocreation process of a mobile application for quantified supervised home-based exercise therapy on PAD patients. The study aimed to design a solution adapted to users' needs, which collects the necessary information for the therapy supervision by health professionals. The users' behaviour with the application allowed the assessment to a set of limitations and potential sources of noise in the supervision data that suggest the evolution to a pervasive solution, by minimizing, or even eliminating, the interaction with the users. The developed tool is a first step towards the creation of a technological ecosystem for the prescription of supervised therapeutic physical exercise, which leverages self-care and allows access to this type of therapy to the entire population. Cardiovascular disease represents a considerable economic burden to society, therefore effective preventive measures are necessary.
\end{abstract}

\section{Introduction}

Patients with peripheral artery disease (PAD) have a severe impairment of functional ability, namely in walking distance due to muscle ischemia defined as intermittent claudication (IC). The discomfort related with IC contributes to a sedentary lifestyle, decreasing physical fitness level, aggravating cardiovascular risk factors and deteriorating the disease.

Exercise programs are an effective, low cost, lowrisk option compared with more invasive therapies for IC [3]. Structured community- or home-based exercise programs take place in the personal setting of the patient rather than in a clinical setting [8]. Programs are selfdirected with guidance of healthcare providers that prescribe an exercise regimen similar to a supervised program [14]. However, frequently, the patients overestimate the claudication severity including on the Walking Impairment Questionnaire (WIQ), with many reporting distances falling below the walking treadmill test results. In contrast, distances reported through Information and Communication Technologies (ICT) tools (as Google Maps ${ }^{1}$ or Open Street Maps ${ }^{2}$ ) have no such discernible trend, suggesting that landmarks-based method is efficient and allows remote control of patient compliance [1].

The aim of the research work is to assess the needs for the supervision of physical exercise therapy and identify potential limitations of mobile application on the acquisition of data, to ensure effective supervision. To reach this objective, four research questions were defined: (RQ1) What data is necessary for the supervision of therapeutic physical exercise for patients with PAD? (RQ2) Are the sensors on a smartphone sufficient to supervise therapeutic physical exercise for PAD patients? (RQ3) Can patients introduce bias in the supervision data by using an m-health app? (RQ4) Are there technological solutions that can mitigate the potential supervision data bias and ensure effective $\mathrm{m}$ health supervision?

This paper introduces the cocreation approach followed for the development of a supervised home-

2 https://www.openstreetmap.org/

${ }^{1}$ https://maps.google.com 
based exercise therapy mobile application for claudicant patients. The app monitors the exercise by controlling the quality and quantity of walking, enabling individuals to perform the recommended exercise program autonomously, empowering self-monitoring as a key to changing long-term behaviour. A cloud-based system aggregates the data collected during the walk, enabling the exercise therapy supervision by making it accessible to the medical doctors, health exercise professionals and therapists, who follow the patient program. The collected data includes the full characteristics of the exercise dose accomplished, thus allowing to follow up the patient evolution.

The cocreation process, with the collaboration of the entire community, including patients, doctors, health and sport professionals, aimed at the RQ1 challenges, and had impact on the application's high-fidelity prototype. The evaluation of the accuracy of the sensors on the smartphone and the development of algorithms to improve the quality of the data, contributed to RQ2. The tests carried out with PAD patients in a real environment, showed situations in which users deliberately or inadvertently introduce bias in the supervision data (RQ3). The discussion of the results demonstrated the need to evolve towards a pervasive application, which minimizes or even eliminates the interaction with the PAD patients (RQ4). The major contribution of this paper is the identification of the bias sources for the remote supervision of therapeutic physical exercise for PAD patients, the proposal of possible ICT solutions for its mitigation and the roadmap that can lead to an ICT based ecosystem to the prescription of therapeutic physical exercise for PAD patients.

This paper is structured in 8 sections: the first section, the current one, contextualize the problem and provides the research objectives of this work. The background section, presents the concepts related with this work, including peripheral artery disease and the current state of the art on activity tracking apps and devices. Section 3 introduces the research methodology that is being followed, defining the iteration cycles and macro phases of the development. The system architecture is presented in section 4 , followed by the detailed description of the prototypes development process in section 5. Section 6 presents the evaluation, followed by the discussion on section 7 , identifying the limitations, lessons learned and the contributions to theory and practice. Finally, the last section closes up with some final remarks.

\section{Background}

Cardiovascular disease (CVD) remains the most common cause of death worldwide, with the 2013
Global Burden of Disease study estimating that CVD caused 17.3 million deaths globally. It accounted for $31.5 \%$ of all deaths [23]. PAD is a manifestation of systemic atherosclerosis affecting $12-20 \%$ of men and women older than 55-65 years [6]. It has been calculated that about 27 million people in Europe and US suffer from this pathology, representing a relevant socioeconomic problem in western countries [21]. Because of its high prevalence, high rates of nonfatal cardiovascular ischemic events, increased mortality, and diminution of quality of life are the significant consequences of PAD in US communities. With a conservative estimate of $>202$ million affected with this disease globally, a recent meta-analysis of 34 studies analysis showed a relative increase in PAD prevalence of $23.5 \%$ during the first decade of the new millennium [3].

\subsection{Peripheral artery disease (PAD)}

PAD results of atherosclerosis in the lower limb arteries. The disease can cause intermittent claudication, limb ulceration, infections and amputation. Patients usually have limited walking abilities, both regarding duration and speed, with impact to their life quality [1]. This scenario is particularly important due to its relationship with ageing and its well-known cardiovascular risk factor and cardiovascular diseases [18]. Therefore, the disease will continue to grow as the global world population is ageing, with increasing amount of resources required [3].

Supervised progressive physical exercise significantly improves functional capacity, improving the walking distance and reducing the mortality and cardiovascular events in patients [1][18]. However, several factors contribute to the decrease of the ability to effectively engage in physical activity, including ageing. Therefore, active ageing with participation in physical activity is a key factor to fight the disease [18].

In the last decades, several strategies to introduce technology for ensuring the regular practice of physical exercise for PAD patients have been studied by the scientific and medical community, as previously presented in [25]. Recently, the use of technology has arisen in this domain. Gernigon et al. [5] performed in 2014 the first clinical applicability of a global positioning system (GPS) to monitor walking abilities of PAD patients. More recently, Khambati et al. [8] have tested the accuracy of GPS based mapping tools to become a measurement of walking distance in PAD patients. The authors used Google Maps as a GPS based mapping tool, and the results showed that: "it offers an accurate, efficient, inexpensive, and readily accessible way to assess walking distances in patients with $P A D^{\prime \prime}[8]$. Shalan et al. [22] proposed YORwalK, a mobile Application for people with IC to promote exercise and track changes in walking ability in this 
population. The application follows the design of current activity tracking apps, adapted to the walking capabilities of the PAD patients. The application is compared with Stanford VascTract [18] that aims to collect data for research studies.

\subsection{Activity tracking apps and devices}

Currently there are several applications and devices that can be used to track fitness and users' activities.

Major application stores have a special category for Health and Fitness apps. Among the most downloaded and top applications of Google Play store and Apple AppStore are: Strava ${ }^{3}$, Nike+ Run $\mathrm{Club}^{4}$, Runtastic ${ }^{5}$ and Samsung Health ${ }^{6}$. Google and Apple have their own applications for fitness that are usually pre-installed: Google Fit and, Apple Health and Activity. These applications share the same concept: track users' activity. Most of them include interfaces that allow users to record exercises, measure the distance walked/run including the altimetry and show burned calories. Each application has its own social network for users to share their achievements. Moreover, integration and data interchanging among applications is possible, usually, using GPS eXchange Format (GPX) and Training Center XML (TCX) format.

Fitness applications are not restricted to smartphones. There are also specific devices and applications for wearables, as smartwatches, that allow to extend the apps features to these devices. Specific smartwatches, also known as fitness watches, have embedded firmware with applications for multi-sports. Among the most well-known devices are Garmin ${ }^{7}$, Polar $^{8}$, Fitbit ${ }^{9}$, TomTom ${ }^{10}$ and Suunto ${ }^{11}$. Regarding the hardware features, some of these devices have built in GPS sensor that allow the tracking of outdoors activity, and also accelerometer and gyroscope, that together, act as a pedometer and allow measuring the walking distance indoors. Another feature that some of these devices also include is the heart rate sensor, built in or external (as a hearth rate band). On the other hand, generic smartwatches can perform the same features using build in apps. These apps are often extensions of the current available apps for the smartphones, allowing its usage in the smartwatch. The features available depend on the hardware of the device, that often includes built-in GPS, accelerometer, gyroscope, barometer and heart rate monitor. Popular devices are

\footnotetext{
3 https://www.strava.com

4 http://nikeplus.nike.com/

5 https://www.runtastic.com

6 https://health.apps.samsung.com

7 https://www.garmin.com/en-US/
}

the Apple Watch, the Samsung Gear, and the Android Wear devices.

A recent study [23] presents the requirements and preferences for mobile health intervention in PAD patients and their therapist, that cannot be fulfil by generic activity tracking apps or devices. Specific requirements underly the need of specific applications for this kind of intervention. PAD apps need to ensure the privacy of the patients and support the community, including family, caregivers, medical doctors, therapists, physical exercise experts, and physiologists, providing different access levels to the information. The PAD apps should also be able educate the patient to adhere to physical treatment, and to understand the evolution of the disease. It should also be noted that current generic (medical) tracking apps are developed to operate in asynchronous mode, recording patients' data and subsequently allowing access to information. For PAD patients, real time advise to stop exercise with extreme pain levels can have impact in the treatment program and the evolution of the disease.

\section{Methodology}

The development of the pervasive monitoring platform for PAD patients followed a Design Science Research methodology [8], associating the principles of user-centred development defined by ISO 9241-210: 2019 [8] to the design cycles. The process involves active participation of experts and users in the development stages, to specify and refine the requirements of a solution suitable for the users' needs and their digital literacy. This approach allowed achieving a strong relationship between the research team and potential users, clearly identifying their habits, requirements and needs of the particular group being studied. This approach aims the development of adequate solutions for users, respecting their digital literacy and having minimal impact on their habits, with the purpose of promoting the objectives of the defined plan of physical exercise practice for PAD patients. Therefore, the Lukosch et al. [13] method was applied, combining the development of low and high-fidelity prototypes and participatory design methods. The main advantage of this methodology is shorter and faster return cycles and, consequently, reducing the risk associated with the development process [17]. The

\footnotetext{
8 https://www.polar.com/

9 https://www.fitbit.com/

10 https://www.tomtom.com/sports/fitness-trackers/

11 http://www.suunto.com
} 
process ended up with the generation of a functional prototype. This sequence of evaluation, specification and development is defined as an iterative cycle of the methodology, being repeated when redefined needs and functionalities for the system, not contemplated in the generated functional prototype.

The systematization of the process establishes four macro phases for the methodology iterative cycle (Figure 1):

(M1) Requirements and specification;

(M2) Low fidelity prototypes;

(M3) High fidelity prototypes;

(M4) Functional prototype.
The involvement of experts and the target groups in the development of the system aimed at the successive refinement of specifications, meeting the needs of both groups. This strategy seeks to have input from the stakeholders throughout the process. The participants of both groups followed the all development cycle (M1)-(M4).

The expert group includes two researchers from the domain of sports sciences both female from age groups 20-25 and 35-45. The experts have more than 2 years' experience following PAD patients. One of the experts is a senior researcher with more than 15 years in physiological sciences.

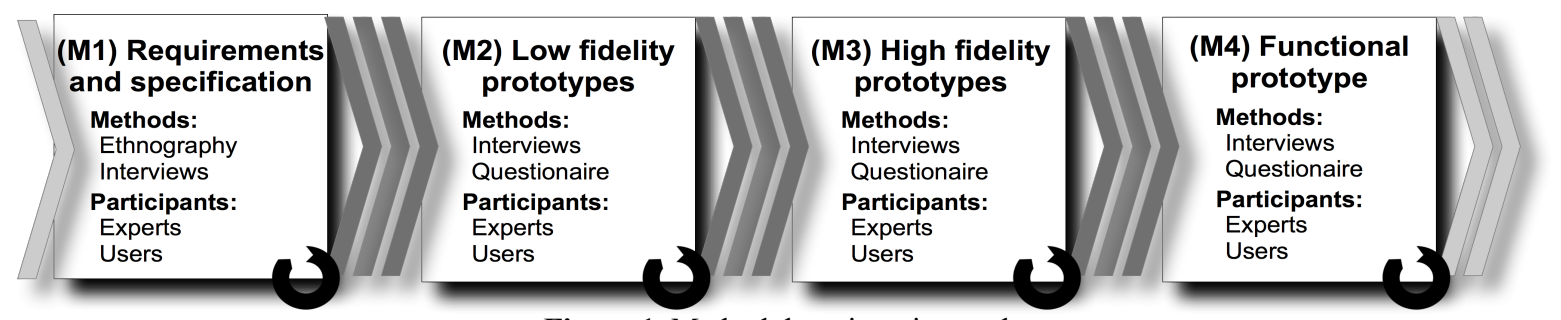

Figure 1. Methodology iterative cycle

In (M1) a short ethnographic study was applied to identify patients' habits. This study took place for two weeks and aimed to follow up the patients' exercises, noting their habits, usual objects and use of technologies. The study was the basis for the script of semi-structured interviews that were carried out with the patients in order to identify the usability requirements of the system. Other features were identified through interviews with experts, including researchers and clinicians in the domain of PAD. The identified functionalities and requirements were classified with a priority degree that was considered for inclusion in the development of each iterative cycle of the methodology.

The macro phases (M2), (M3) and (M4) consist of micro-cycles, in which expert and user evaluations were carried out. The micro-cycles have as stopping condition the approval by the experts and the achievement of an average degree of satisfaction in the usability evaluations with users using the Lewis usability questionnaire [12] higher than $85 \%$. In each of the evaluations, expert and user groups will consist of five representative actors as defined by in [14][16].

\subsection{Participants}

The development phase involved experts and PAD patients' groups. The development monitoring group intends to be representative of the target population, ensuring the agility of the process. Therefore, a restricted set of experts from the target groups were selected, with availability and the ability to support researchers throughout the development process.
The PAD patients group has two participants, male, over 65 years old. They participate in a PAD exercise program at a local hospital, being part of a group that performs exercise three times a week in a treadmill, monitored by the experts that are part of the expert group.

\section{System Architecture}

The high cost of the hospital supervised exercise program requires the development of a solution that allows the engagement and participation of more patients. The results of the program are aligned with those reported in the literature, reporting very positive results for the patients. Therefore, an ambulatory, low cost program will allow its expansion to a wide range of PAD patients.

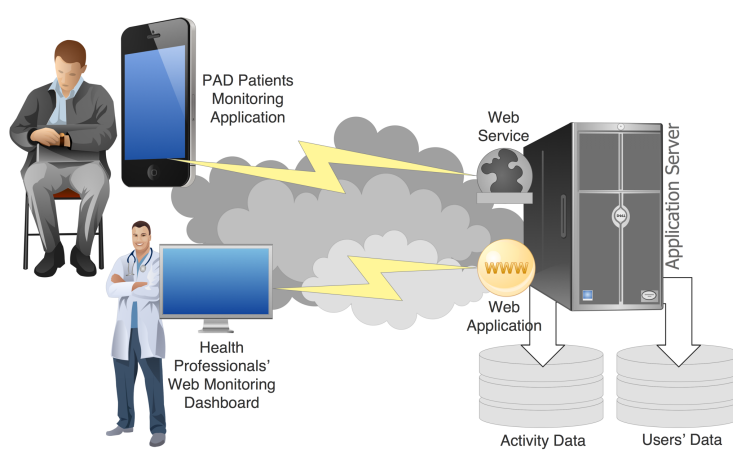

Figure 2. System Architecture

To implement the program, a pervasive monitoring platform for PAD patients is being developed. The 
proposed system is a client-server solution, based in a pervasive monitoring application connected to an application server, through a web service. The web service allows the central collection of data from the PAD patients applications. The data is stored in a database and can be visualized by the health professionals' through a web application. The overall system is presented in Figure 2.

The system was designed in order to require minimal maintenance, easy replication and scalability. The application server hosts a web service developed in PHP and a web application developed in Laravel (a PHP Web development framework). The collected data is stored in a database (Activity Data) separated from the patients' data (Users' Data). This separation allows the anonymization of data and ensured patients privacy. Moreover, the application server also ensures this detachment, providing two separate web applications: one that provides users' activity data; and another that provides patients' profile data. A federated authentication system allows the access to both applications and the data fusion in the client application for visualization by authorized users.

The PAD Patients Monitoring Application is a mobile application that have been developed using a user-centered design. The application allows the patients to record the exercises and send the collected data for health professional supervision. Its operation is based in a state machine, as showed in Figure 3.

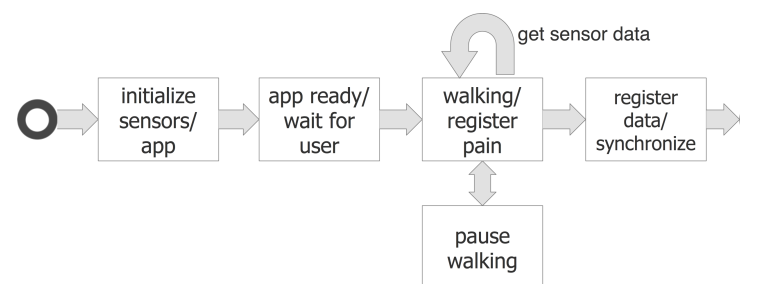

Figure 3. PAD Patients Monitoring Application flow

The development of the several prototypes of the PAD Patients Monitoring Application is described in the next section.

\section{Application prototypes}

The development of the application followed 4 cycles defined by the methodology. The development cycles aimed to refine the versions of the prototype, in close collaboration with experts and users.

For the first implementation of the application the mobile environment was chosen as it provides a more flexible solution for exploring the interaction, data collection and sensoring features required for patient exercise monitoring. A mobile based solution can be easily ported/extended to the smartwatch, or other devices, after analysing the conditioning and interaction needs. In a first approach, we need to understand how receptive and technologically the users are. Users are familiar with smartphones, as they are already part of their daily lives. The introduction of the smartwatch, or other devices, will be progressive and possible in a second phase, when refined and gauged the real potentialities of information inference, as well as minimized the user interactions.

The first development cycle (M1) took place in May 2019 and had the objective of surveying application requirements. In this phase, the patients of the PAD

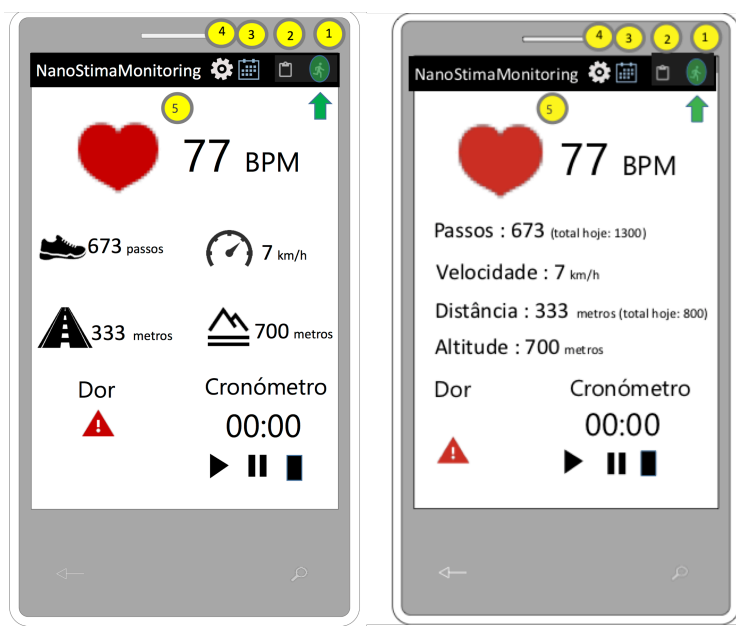

Figure 4. Low fidelity prototypes: text (right) and symbols (left) versions. Translation "Portuguese"-

English: "passos"-steps; "velocidade"-speed;

"distancia"-distance; "dor"-pain; "cronometro"-timer; "metros" - meters; "hoje" - today;

patients' group were followed in their daily exercise plan. The follow-up of the PAD patients was performed inside the hospital area, outdoors, in a determined route for the purpose. Monitoring was carried out by researchers in sports sciences who normally follow the patients, collecting a set of data previously defined by the research team, including daily habits and personal objects used during the walk (mobile phone, music player, radio, clock, etc). The second author also participated in the observations. In addition to this information collection, semi-structured interviews were carried out with the expert group.

The information collected allowed the definition of the requirements of the application and their classification, in order to delimit the consequent development phases. Among the requirements identified, the most important were: track record, total distance travelled, slope, time in motion and time at rest, level of pain, and speed. It should be noted that the temporal correlation of some of these indicators are also requirements of the application, such as the evolution of pain along the walk and its relationship with the stops. 


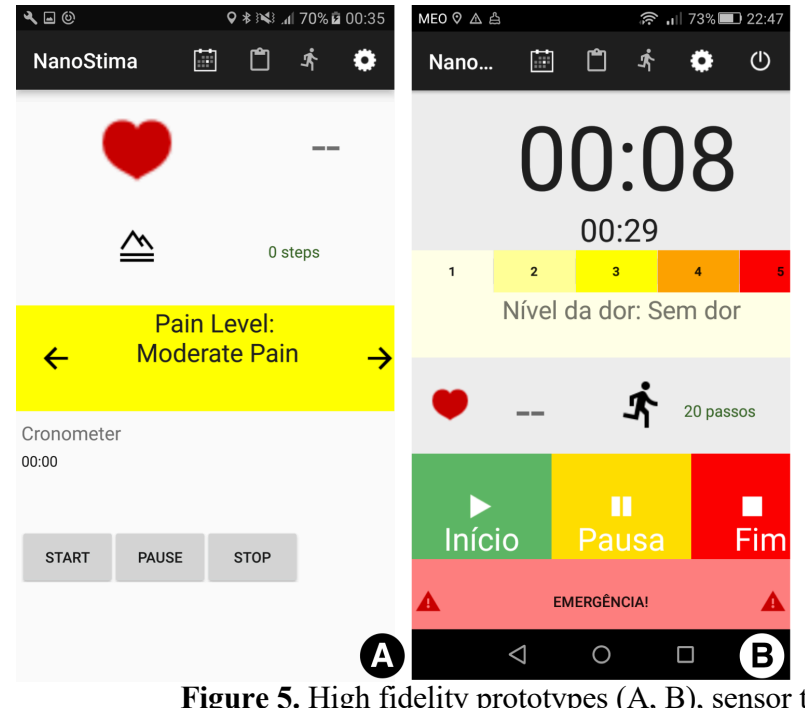

Figure 5. High fidelity prototypes (A, B), senso

The requirements identified in the first development cycle allowed the creation of low-fidelity prototypes of the application (Figure 4). These prototypes incorporate the needs identified in step (M1), providing interface features as initiate walking, pausing and recording pain during the course to the user. Associated with these commands is also a set of information that can be visualized by users. Two alternatives have been proposed: textual description and the use of symbols. The low-fidelity prototypes were presented to users and experts at work meetings held in July 2019. The result of these meetings allowed us to refine low-fidelity prototypes developed in particular at the level of pain level registration. Pain follows a simplified 5 level scale: no pain; mild; moderate; severe; maddening. The scale has associated colours, from green (no pain) to red (maddening). The implementation of the prototypes was accomplished in the third cycle of the development methodology.

The development of the high-fidelity prototype and the functional prototype of the application followed an agile process, including several sprints. The first sprints aimed the development of the graphical interface, for its validation with the patients and experts' groups as a high-fidelity prototype. Two prototypes have been developed and discussed, as presented in Figure 5.

The first developed high-fidelity prototype follows the low fidelity prototypes, with a simplified interface, small buttons and including a wide area for definition of pain level, the key feature of this application. The tests to the development of the prototype performed by the experts and patients' group revealed that the interaction mode with the designated area to indicate the level of pain was not adequate. The identified problem is that it does not present all the possible options, forcing the user to discover the options by selecting them. In addition,

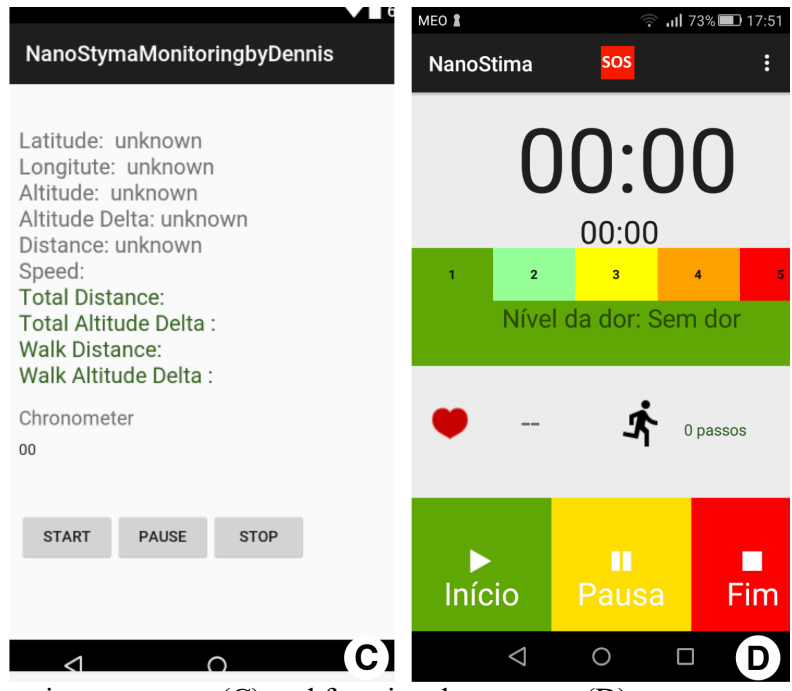

switching to another option automatically $\operatorname{logs}$ a different level of pain, which misleads by introducing many false positives into the collected data.

The collected feedback allowed to evolve to a second prototype, shown in Figure 5-B. The acceptance of this prototype by the users was more positive than previous version (Figure 5-A). Of the few aspects to be improved was the location of the help button, which was sometimes used unnecessarily (due to its location at the bottom of the screen), and the contrast of colours in the levels of pain, which, in situations of high light, are difficult to see. These aspects were refined in the functional prototype (Figure 5-D).

Parallel to the development of high-fidelity prototypes, a prototype was developed to test sensors and functionalities. This prototype was intended to test the accuracy and APIs of access to the different sensors of the device, and to assess its ability to respond to the application requirements. The prototype gathered data from the GPS, accelerometer, gyroscope, magnet and barometer. The prototype text interface (Figure 5-C) allowed the research team to analyse the data collected from the sensors. Following a separation of concerns architectural approach, for the prototype development allowed a rapid integration of the backend with the user interface, and fosters the app future (agile) evolution.

The functional prototype was brought out from the merge of the high-fidelity prototype and the sensor test prototype. Thus, the mobile device sensors functionalities and capabilities have been integrated into an application logic layer. This layer implements the functionalities that are presented to the user by the interface. The multi-layer model allows future exploration and migration to other devices of the functionalities, adapting the interface and/or the sensors to the new environment. 

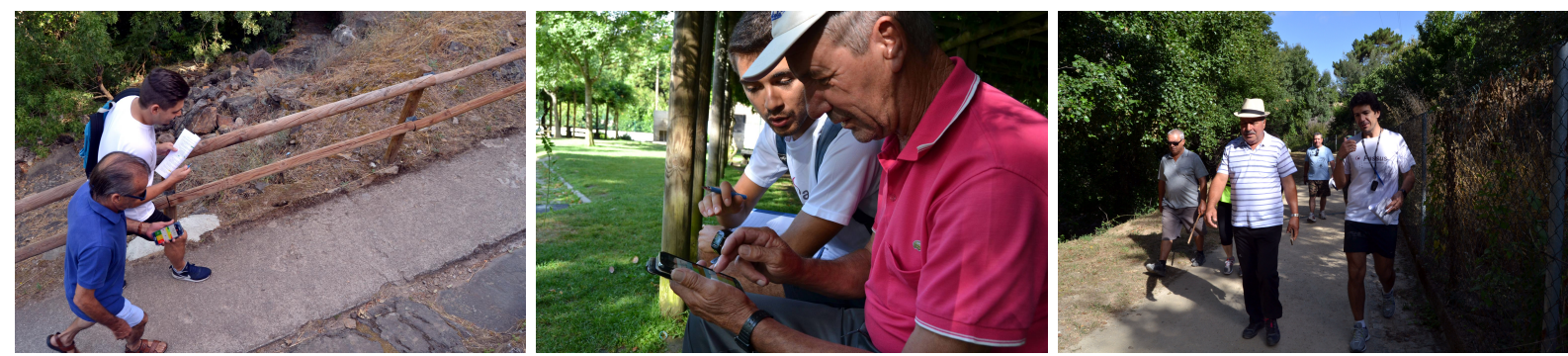

Figure 6. Evaluation event, help at Corgo Park, Vila Real - Portugal (September 2019)

\section{Evaluation}

The prototype was tested by a group of PAD patients at an event organized by the research team. The event was attended by 5 patients, some of which accompanied by a family member, and 5 researchers. None of the participants involved in this trial had had contact with the application before. The objectives of the trial were: the evaluation of the users' satisfaction with the developed prototype; the measuring of the users' interactions with the application; and the collection of observation data on the use of the application during the walk.

The event took place on a Saturday in September 2019, at 9 am. in Corgo Park in Vila Real, Portugal, a place with several pedestrian paths, accessible for this type of patients (Figure 6). The script for the trial was defined as:

- Reception of participants

- Briefing: application features;

- Trial groups: assignment of a researcher to each patient, to follow the exercise and take notes about the usage of the application;

- 40 minutes' walk;

- Evaluation questionnaire;

- Wrap up, food and drinks.

Analysis of the questionnaires reveals that the participants are in a 59-66 age group. Only one of the patients had previous experience with smartphones. The global opinion is satisfaction with the experience of using the application and the desire to continue using it. One of the participants revealed that he had difficulties interacting with the application. There was a total of 3 requests for help from researchers during the experiment. An interaction counter was included in the application to count the number of clicks/touches in each of the interactive elements. The results are summarized in Table 1.

Each of the researchers who followed the patients took notes on the use of the application along the route. The notes share the following relevant topics:

- Patients are interested in understanding and using the application;
- At the beginning, users start to walk and see the data that is made available in the application, in particular the elapsed time, the number of steps and their relationship with the distance covered;

- After the first few minutes (1-3 minutes) of walking, users turn off their smartphone and there is no further interaction with the app;

- In some cases, the researchers asked the patients if they feel pain, and, at that moment, they opened the application and registered an increase in the pain level;

- There were no interactions without active intervention of the researcher, not even stopping the activity, which, in all cases, had to be reminded by the researcher;

- In a specific situation, there was a technical problem with the application, that was only detected at the end of the exercise, because the user did not use the application (PAD_4). The walk data was not recorded.

Table 1 - Patients' interactions with the mobile app during the exercise

\begin{tabular}{|c|c|c|c|c|}
\hline \multirow{2}{*}{$\begin{array}{c}\text { User } \\
\text { ID }\end{array}$} & \multicolumn{4}{|c|}{$\begin{array}{c}\text { Interactions with app buttons for } \\
\text { successfully execute the task (\#clicks) }\end{array}$} \\
\cline { 2 - 5 } & start & stop & pause & pain level \\
\hline PAD_1 & 2 & 1 & - & 1 \\
\hline PAD_2 & 4 & 1 & - & 2 \\
\hline PAD_3 & 2 & 2 & - & 4 \\
\hline PAD_4 & 1 & 1 & - & - \\
\hline PAD_5 & 1 & 1 & - & 1 \\
\hline
\end{tabular}

\section{Discussion}

The major objective of the work presented in this paper is to identify the data requirements for supervising physical exercise therapy for PAD patients through a mobile application; evaluate the feasibility of using a mobile phone to collect the data; and identify limitations and pitfalls that can be introduced by the patients interaction with the system.

The codesign of the application allowed to identify the major requirements for the supervision of the physical exercise therapy, that include: the GPS tracking 
of the walks, walking time, number of stops, and pain level (RQ1). Some of this data can be retrieved from the mobile phone sensors; other can be calculated with the data retrieved from the sensors (as the number of stops, or the number of steps); but there is some data that requires the user's interaction (pain level) (RQ2).

The evaluation revealed that the user interaction with the application are minimal, in some cases, just to start the recording of the exercise. There is a high impact of this behaviour in the data quality (RQ3), and in the capability of the application to provide the required data for therapy supervision. Current practice of supervised exercise requires that the therapist register the patients' pain level each 5 minutes, asking for his/her input. However, this feature is not used in the mobile app, as the interaction is very restricted. Therefore, further development is required in order to identify if it is possible to pervasively extract pain level from the data gathered from mobile phone sensors (RQ4). Empirical observations show differences in the gait pattern of patients as pain increases, forecasting future research in this field.

The presented research follows a holistic approach of the physical exercise therapy for PAD patients, providing perspectives to each stakeholder: patients and health and physical exercise professionals. The proposed system allows health and physical exercise professionals to prescribe physical exercise that can be supervised with the help of the presented PAD application. Patients' can track their exercise prescription and be remotely followed by the health and physical exercise professionals. This perspective distinguishes this approach from others found in the literature. Stanford VascTract [18] focus only on the data collection for research proposes. YORwalK[22], on the other hand, aims to provide a tool to empower patients, without the real-time follow up by the clinical team. However, the results show limitations and challenges in bringing together the requirements and needs of both PAD patients and health and physical exercise professionals.

\subsection{Limitations and lesson learned.}

The major limitation identified in this study is the lack of interaction of patients with the mobile application. This absence of inputs from the user may comprise the capability of the system to supervise the physical exercise therapy. An example of this is the information about the pain level during the exercise, in particular the moment when the pain starts. This information is important to evaluate the progress of the disease, and therefore must be acquired by the supervision system and provided to the medical doctors.
Another identified limitation was the need to manually synchronize data with the central system. As the patients have a low interaction with the application, the synchronization of data with the central system is not a priority and must therefore be automated.

The close relation between the research team, the experts and the PAD patients during the cocreation process allowed to go further in the possible pitfalls in the supervision. A common situation is patients avoiding therapy, not only physical but also pharmacological. A remote supervision system, without strong authentications mechanisms can be easily bypassed by patients to avoid therapy, as for example asking a family member to perform the walk on his/her behalf.

The limitations identified in the current solution for the supervision of physical exercise therapy for PAD patients, endeavours future research lines that aim a fully automated monitoring tool, without requiring users' input to accurately ensure the supervision. Major lesson learned with this study include:

- Cocreation process allowed the definition of the requirements for the supervision of therapeutic physical exercise, but did not predict the patients' behaviour with the app.

- The low aptitude of this population for the use of smartphones constrains the interaction, with impacts in the data gathering.

- Patients' unwillingness to adhere to therapy can lead to divergent behaviours and misuse of the application, requiring an activity oriented patient authentication, to ensure authenticity of data collected.

- Using a pain level scale requires training the users to distinguish each level. Empirical observations revealed that pain has impacts in the gait patterns of PAD patients. Mechanisms for automatic recognition of pain level based on gait patterns can be explored to ensure a faster enrolment of patients without the need of specific pain level training.

- The physical exercise therapy concept should follow same prescription procedures of pharmacological therapy, with evidences for the patient of the need to perform the therapy. A formal prescription, following the usual clinical treatment procedure, can have benefits in the patient adherence to the therapy.

The number of participants in this study can also be considered a limitation. The development process followed a user centred approach, with a advisory board of two researchers, experts in PAD, and two PAD patients. The evaluation was performed with a PAD patients' activity, for one day. Five PAD patients participated in the evaluation. Despite the low number of participants, results show a high potential of the 
application, requiring further development in order to address the previously identified limitations.

\subsection{Theoretical and practical implications}

The lessons learned during the user centred approach applied for developing this prototype showed that the best option would be the full autonomous monitoring, without user interaction. User interactions can be performed in order to visualize selected information on demand. Therefore, the research team planned an approach to progressively reduce the number of interactions with the user and move towards a nointeraction solution. This approach is already followed by some market applications and devices to monitor physical activity (cf. section 2.2), however without the same precision and requirements that we have in the specific case of PAD. First, we need to detect when the user is moving or is stationary. The start of an exercise always requires the intervention of the user to explicitly declare the start of the session and the movement record associated with that session. Depending on the type of user, and their habits, this process can be simplified, or even suggested based on contextual information, predicting the activity. If the user has a routine followed regularly, the application can easily detect the beginning of the exercise, suggesting the user to confirm it. The routine can be inferred through the daily practices of the user or based on their records in the agenda or even another type of activity planning application (which can even be integrated into the application).

Another challenge is related to pain level. A first approach can suggest that the severe pain level is associated with a stop walking event, which can be easily detected by the device sensors. But there is not a direct relation. Usually, patients stop when they feel severe pain as they are forced to stop walking. However, there are other situations, such as finding a friend and stopping for a conversation. In this situation, a false positive can be detected, in which a stop is associated with severe pain without happening. So, there is a need of a user confirmation of this type of situations. However, the gait analysis can provide very interesting data about pain level. The relationship between severe pain and stop walking can be inferred through the gait pattern. As the pain increases, the patient's walking speed decreases, forcing him to stop when it becomes severe. Thus, it becomes possible to infer the various levels of pain based on the analysis of patient gait patterns. This process requires learning based on interactions with the user, so that an association between a pattern, a speed and a certain level of pain can be made. Current work of the research team already has promising results on this topic [19].
One of the major challenges is to ensure a strong patient authentication to make sure that is the real user that is performing the therapy remotely. This ensures an effective supervision of the physical exercise therapy. Using on device authentication, based on biometric data is not a solution, as the patient can authenticate and deliver the device to a third party to perform the exercise. Therefore, what is required is a method that can validate the exercise with the person. The research work carried out for automatic pain level recognition based on gait patterns raised a research question aiming to find evidences that each $\mathrm{PAD}$ patient has a specific gait pattern, a gait fingerprint. Current state of the art [3] provides promising evidences that biometric gait recognition can be used to authenticate PAD patients and ensure that each exercise is performed by the patient.

\section{Final remarks}

This paper presented an exploratory approach for the implementation of a holistic physical exercise therapy system for PAD patients, that can bring together the needs of patients and health professionals. The objective of the presented research work was to find evidences that remote supervision of physical exercise therapy through a mobile application is possible and feasible, identifying the requirements and challenges to ensure an accurate system that can be used in real world scenarios.

The development process of a mobile application for monitoring physical exercise of PAD patients, using a cocreation approach, was presented. A functional prototype was implemented following the gathered requirements and evaluated with a group of PAD patients.

The evaluation has promising results, but also raised some limitations that required further research to study solutions to overcome the patients' behaviours and ensure gathering clean supervision data. The discussion of the results and the practical implications of the identified limitations endeavours the creation of a full autonomous application, capable of monitoring PAD patients without requiring user interaction. As discussed, several challenges remain in order to ensure the full autonomy of the solution, including the inferring data such pain level or user authentication through gait patterns analysis. Moreover, patients' motivation to adhere to physical exercise treatment can be enhanced with the use of gamification and socialization strategies [26]. Further users' studies are required for validation of the solution with larger groups of patients, representing a broader target population. 


\section{Acknowledgments}

This work is financed by the ERDF through the NORTE 2020, under the PORTUGAL 2020 and by National Funds through the Portuguese funding agency, FCT within project Ref: NORTE-01-0145-FEDER031161- PTDC/MEC-VAS/31161/2017

\section{References}

[1] Abaraogu, U. O., Abaraogu, O. D., Dall, P. M., Tew, G., Stuart, W., Brittenden, J., \& Seenan, C. A. (2020). Exercise therapy in routine management of peripheral arterial disease and intermittent claudication: a scoping review. Therapeutic Advances in Cardiovascular Disease, 14, 1753944720924270.

[2] Aggarwal, S., Moore, R.D., Arena, R., Marra, B., McBride, A., Lamb, B., Martin, B.J., Stone, J.: Rehabilitation therapy in peripheral arterial disease. Canadian Journal of Cardiology 32(10), S374-S381 (2016)

[3] Boyd J.E., Little J.J. (2005) Biometric Gait Recognition. In: Tistarelli M., Bigun J., Grosso E. (eds) Advanced Studies in Biometrics. Lecture Notes in Computer Science, vol 3161. Springer, Berlin, Heidelberg

[4] Conte, M.S., Pomposelli, F.B., Clair, D.G., Geraghty, P.J., McKinsey, J.F., Mills, J.L., Moneta, G.L., Murad, M.H., Powell, R.J., Reed, A.B., et al.: Society for vascular surgery practice guidelines for atherosclerotic occlusive disease of the lower extremities: management of asymptomatic disease and claudication. Journal of vascular surgery 61(3), 2S-41S (2015)

[5] Gernigon, M., Le Faucheur, A., Noury-Desvaux, B., Mahe, G., Abraham, P., Group, P.G.S.C.: Applicability of global positioning system for the assessment of walking ability in patients with arterial claudication. Journal of vascular surgery 60(4), 973-981 (2014)

[6] Giugliano G, Sannino A, Brevetti L, et al. Ankle/brachial index to everyone. BMC surgery. 2012; 12 Suppl 1: S18

[7] Gornik, H.L., Beckman, J.A.: Peripheral arterial disease. Circulation 111(13), e169-e172 (2005)

[8] Hageman, D., Fokkenrood, H., Essers, P. P., Koelemay, M., Breek, J. C., Vahl, A., ... \& Teijink, J. (2019). Improved Adherence to a Stepped Care Model Reduces Costs of Intermittent Claudication Treatment in the Netherlands. European Journal of Vascular and Endovascular Surgery, 58(6), e47.

[9] Hevner, A. R., March, S. T., Park, J., \& Ram, S. (2004). Design science in information systems research. MIS quarterly, 75-105.

[10] ISO 9241-210:2010 Ergonomics of Human-System Interaction - Part 210: Human-Centred Design for Interactive Systems. 2015. [Accessed July 10, 2020]. Available

from: https://www.iso.org/standard/77520.html

[11] Khambati, H., Boles, K., Jetty, P.: Google maps offers a new way to evaluate claudication. Journal of Vascular Surgery (2017)

[12] Lewis, J.R.: Ibm computer usability satisfaction questionnaires: psychometric evaluation and instructions for use. International Journal of Human-Computer Interaction 7(1), 57-78 (1995)

[13] Lukosch, H., van Ruijven, T., Verbraeck, A.: The participatory design of a simu- lation training game. In: Proceedings of the winter simulation conference. p. 142. Winter Simulation Conference (2012)

[14] Machado, I., Sousa, N., Paredes, H., Ferreira, J., \& Abrantes, C. (2020). Combined aerobic and resistance exercise in walking performance of patients with Intermittent Claudication: Systematic Review. Frontiers in physiology, 10, 1538.

[15] Nielsen, J.: Usability engineering. Elsevier (1994)

[16] Nielsen, J.: Usability 101: Introduction to usability (2003)

[17] Pereira, L.L., Roque, L.: Towards a game experience design model centered on participation. In: CHI'12 Extended Abstracts on Human Factors in Computing Systems. pp. 2327-2332. ACM (2012)

[18] Raheel Ata, et al, VascTrac: A Study of Peripheral Artery Disease via Smartphones to Improve Remote Disease Monitoring and Postoperative Surveillance, J Vasc Surg, June 2017, Volume 65, Issue 6, Supplement, Pages $115 \mathrm{~S}-116 \mathrm{~S}$

[19] Renner, K., Filipe, V., Torres-Pereira, L., Silva, I., \& Paredes, H. (2020, October). Gait pattern analysis with accelerometer data from a smartphone in PAD patients. In Proceedings of the 8th IEEE International Conference on E-Health and Bioengineering - EHB 2020.

[20] Schiattarella, G.G., Perrino, C., Magliulo, F., Carbone, A., Bruno, A.G., De Paulis, M., Sorropago, A., Corrado, R.V., Bottino, R., Menafra, G., et al.: Physical activity in the prevention of peripheral artery disease in the elderly. Frontiers in physiology 5, 12 (2014)

[21] Schiattarella GG, Perrino C, Magliulo F, et al. Physical activity in the prevention of peripheral artery disease in the elderly. Frontiers in physiology. 2014; 5: 12.

[22] Shalan, A., Abdulrahman, A., Habli, I., Tew, G. A., \& Thompson, A. (2018, April). YORwalK: Desiging a Smartphone Exercise Application for People with Intermittent Claudication. In MIE (pp. 311-315).

[23] Townsend N, Wilson L, Bhatnagar P, Wickramasinghe $\mathrm{K}$, Rayner $\mathrm{M}$ and Nichols $\mathrm{M}$. Cardiovascular disease in Europe: epidemiological update 2016. European heart journal. 2016; 37: 3232-45

[24] van den Houten, M. M., Spruijt, S., Fokkenrood, H. J., Scheltinga, M. R., \& Teijink, J. A. (2018). User Preferences for Mobile Health Interventions: A Survey among Intermittent Claudication Patients and Their Physical Therapists. Annals of vascular surgery, 46, 249256.

[25] Paulino, D., Reis, A., Barroso, J., \& Paredes, H. (2018, July). Technologies Applied to Remote Supervision of Exercise in Peripheral Arterial Disease: A Literature Review. In International Conference on Universal Access in Human-Computer Interaction (pp. 320-329). Springer, Cham.

[26] Pinho, Anabela; Paredes, Hugo (2019). Gamification and MDA framework: conceptual model to promote motivation in the elderly. In Videojogos 2019 - 11th International Conference on Videogames Sciences and Arts. Aveiro 